\title{
Dihydroxyacetone and Methods to Improve its Performance as Artificial Tanner
}

\author{
Craig G. Burkhart ${ }^{*}, 1$ and Craig N. Burkhart ${ }^{2}$ \\ ${ }^{1}$ University of Toledo College of Medicine, USA \\ ${ }^{2}$ University of North Carolina at Chapel Hill, Chapel Hill, North Carolina, USA
}

\begin{abstract}
Dihydroxyacetone binds to proteins in the stratum corneum of the skin imparting a non-toxic, bronze color to the skin. The resulting pigments are called melanoidins, or brown chromophores. Since the 1980's, improved formulations of sunless tanners are available due to more purified sources of DHA and refinements in the DHA manufacturing process. The color is not removed by simple washing, swimming, or natural perspiration, but it only lasts for 5 to 7 days, as skin cells are continuously being shed. Exfoliation, tape stripping, prolonged water submersion, or heavy sweating can lighten the tan, as these all contribute to rapid dead skin cell pealing. Indeed, patients may opt to reapply the product on a regular basis (such as daily or every fourth day) to maintain the skin color.

Possible improvements in dihydroxyacetone products include addition of perfluoropolyether phosphate to lower the formulation's $\mathrm{pH}$, exfoliation with polyethylene beads prior to treatment, wiping skin with an acidic toner just prior to application, addition of strong antioxidants such as caffeic acid phenethyl ester, using a polymer base, and pretreatment of the skin with amino acids to increase binding sites of DHA to the skin.
\end{abstract}

Tanned skin remains a desirable quest by a large segment of the population in Western countries. Several products are being marketed for that coveted dark skin tone without direct ultraviolet tanning. The list of sunless- or self-tanners includes dihydroxyacetone (DHA), color cosmetics, henna, juglone, bronzing gels, color washes, facial highlighters, bronzing powders, tinted moisturizers, and facial foundations [1]. As DHA is the most common active agent in sunless tanners, and as it is neither toxic nor absorbed through the skin into the body (as it does not penetrate beyond the stratum corneum), this manuscript concentrates on this Food and Drug Administration-approved product.

DHA, also named dihydroxy-2 propanone, is a simple three carbon sugar which is derived from plant sources such as sugar beets and sugar cane by the fermentation of glycerin. It binds to proteins in the stratum corneum of the skin imparting a bronze color to the skin. This skin browning effect is non-toxic. DHA reacts chemically with the amino acid groups, which are part of the protein containing keratin layer on the skin surface. Various amino acids react differently to DHA, producing different tones of coloration from yellow to brown. The resulting pigments are called melanoidins, or brown chromophores. These are similar in coloration to melanin, the natural substance in the deeper skin layers. The mechanism of this artificial tanning is totally different from natural tanning, which is based on the increase in melanin pigmentation secondary to ultraviolet exposure. On passing, this chemical reaction of an amino acid with a reducing sugar is often referred to as a Maillard reaction.

\footnotetext{
*Address correspondence to this author at the University of Toledo School of Medicine, 5600 Monroe Street, Suite 106B, Sylvania, OH 43560, USA; Tel: 419-885-3403; Fax: 419-885-3401; E-mail: cgbakb@aol.com
}

The first DHA sunless tanning lotion appeared in the 1960s. It was made by Coppertone and called "Quick Tan" or "QT", and marketed as an overnight tanning agent. The initial products gave the skin a somewhat unnatural apricot color. It was also associated with as orange palms, streaking and poor coloration. Because of the QT experience, many people today still associate sunless tanning with fake-looking tans.

Since the 1980's, improved formulations of sunless tanners are available due to more purified sources of DHA and refinements in the DHA manufacturing process. These newer products provide a more natural looking color with better fading. As a result of consumer concerns surrounding damage associated with ultraviolet radiation, there has been increased popularity of sunless tanning products.

Today, DHA is the main active ingredient in virtually all sunless tanning skincare preparations. Another product occasionally used in combination with DHA is erythrulose. Erythrulose acts identically to DHA, but the reaction occurs more slowly. Sunless tanning products contain DHA in concentrations ranging from $1 \%$ to $10 \%$. Most drugstore products range from $3 \%$ to $5 \%$, with professional products ranging usually from $5 \%$ to $15 \%$. The percentages reflect the product coloration levels, that being from light to dark. Lighter products are easier to use effectively as less streakiness and unevenness are more easily avoidable; however, multiple applications may be needed to reach the desired skin tone. Higher concentrations of DHA often produce the dark hue sought with one coat. The color change takes only one hour to begin appearing on the skin surface, and continue to darken for 24 to 72 hours, depending on formulation type. As alluded to above, patients can apply the product on ensuing days until the desired color is achieved. In areas of 
thicker stratum corneum such as the palms and elbows, the staining is darker, and this has to be accounted for with one's future applications. On passing, DHA stains hair and nails, but does not stain mucous membranes, as these latter surfaces are devoid of a stratum corneum.

The color is not removed by simple washing, swimming, or natural perspiration, but it only lasts for 5 to 7 days, as skin cells are continuously being shed. Exfoliation, tape stripping, prolonged water submersion, or heavy sweating can lighten the tan, as these all contribute to rapid dead skin cell pealing. Indeed, patients may opt to reapply the product on a regular basis (such as daily or every fourth day) to maintain the skin color.

Currently DHA is formulated into sprays, lotions, gels, mousses, and cosmetic wipes. Professional applied products also include spray tanning booths and airbrush tan applications.

DHA does not damage the skin, and is considered a safe skin coloring agent. It also has been proven to be cosmetically helpful in patients suffering from vitiligo. Contact dermatitis is rarely reported. Most cases of sensitivity are due to other ingredients in the skin product preparation, such as preservatives, plant extracts, dyes or fragrances.

DHA offers limited ultraviolet B sunscreen effect, providing a SPF of 3 to 4 . Additionally, melanoids also absorb in the ultraviolet A range as well $[2,3]$. This sun protection, however, is operative only for the first few hours of its application, and does not continue for the duration of the color change.

\section{POSSIBLE IMPROVEMENTS IN DHA PRODUCTS}

DHA is best formulated at a $\mathrm{pH}$ of 4 to 5 which allows for maximum stability and shelf-life. A perfluoropolyether phosphate has been developed which allows for a lower $\mathrm{pH}$ for DHA without increasing skin irritation [4]. By having the skin $\mathrm{pH}$ also of this acidity, a more natural tan appearance can be achieved. Thus, better results can be gained by avoiding alkaline soaps or detergents prior to DHA application. Moreover, wiping the skin surface with a hydroalcoholic, acidic toner just prior to applying DHA will also improve results [1].
The addition of strong antioxidants such as caffeic acid phenethyl ester in DHA formulation allows for a very close approximation of skin tone to the color of natural suntan [5]. This has been studied in respect to color measurements obtained with the Minolta Chromameter and changes in reflectance [5].

DHA would be an excellent candidate for application in a polymer base. Polymers facilitate transport and release of cosmetic and pharmaceutical molecules at desired rates and desired sites. Such a delivery system could better control pH, temperature, and ionic strength of the system. Polymers would allow deposition of more product to the outer skin layers.

Pretreatment of the skin with amino acids would also increase binding sites of DHA to the skin and enhance the color produced. Application of a sulfur-containing amino acid such as methionine sulfoxide has been advocated [6]. Lysine, glycine, and histidine have also been shown to react rapidly with DHA [7].

It is helpful to remove the outer dead skin layers prior to application. This allows deeper penetration of DHA. Because high hydration decreases DHA effectiveness, exfoliation can be achieved physically with an abrasive scrub containing polyethylene beads, a textured cloth, or a hydroxyl acid moisturizer [6].

\section{REFERENCES}

[1] Levy SB. Cosmetics that imitate a tan. Dermatol Ther 2001; 14: 215-9.

[2] Fusaro RM. Melanoidins-sunscreens: dihyroxyacetone with or without naphthoquinones. J Am Acad Dermatol 2009; 60: 164.

[3] Johnson JA, Fusaro RM. Protection against long ultraviolet radiation: topical browning agents and a new outlook. Dermatologica 1987; 175: 53-7.

[4] Pantini G, Ingoglia R, Brunetta F, Brunetta A. Sunless tanning products containing dihydroxyacetone in combination with a perfluoropolyether phosphate. Int J Cosmet Sci 2007; 29; 201-9.

[5] Muizzuddin N, Marenus KD, Maes DH. Tonality of suntan versus sunless tanning with dihydroxyacetone. Skin Res Technol 2000; 6: 199-204.

[6] Draelos ZD. Self-tanning lotions: are they a healthy way to achieve a tan? Am J Clin Dermatol 2002; 3: 317-8.

[7] Nguyen BC, Kochevar IE. Factors influencing sunless tanning with dihydroxyacetone. Br J Dermatol 2003; 149: 332-40. 